\title{
LAS AMETROPÍAS: REVISIÓN ACTUALIZADA PARA MÉDICOS NO OFTALMÓLOGOS.
}

THE AMETROPIAS: UPDATED REVIEW FOR NON-OPHTHALMOLOGISTS PHYSICIANS.

\author{
Virgilio Galvis ${ }^{1,2}$, Alejandro Tello ${ }^{1,2}$, Oscar Blanco', Andrea N. Laiton ${ }^{1,2}$ Marlon R. Dueñas ${ }^{3}$, \\ Priscila Alexandra Hidalgo ${ }^{3}$
}

\section{Resumen}

Los defectos de refracción se originan por un desacople entre el poder de convergencia de los lentes del ojo por un lado, es decir la córnea y el cristalino, que hacen que los rayos que llegan al ojo formen un foco y generen una imagen, y por otro lado la retina, que es la pantalla biológica fotosensible donde esa imagen será transformada en un impulso nervioso. Estos defectos incluyen la miopía, la hipermetropía y el astigmatismo. La presbicia se puede considerar también un defecto refractivo, pero de características especiales, pues solo afecta la visión próxima en mayores de 40 años. Al alterar la calidad de la imagen enfocada en la zona mas sensible a la luz de la retina (la mácula), disminuyen la agudeza visual. Para su manejo existen desde el uso de anteojos y lentes de contacto hasta la corrección quirúrgica (cirugía refractiva). La incidencia de ciertos errores de refracción (específicamente de la miopía) se ha incrementado en la últimas décadas, y se han identificado algunos factores ambientales relacionados con ello. Algunas medidas de manejo médico de la misma han mostrado un efecto positivo en el control de su aparición y progresión.

Palabras Claves: Errores de refracción; presbiopía; miopía; hiperopía; astigmatismo.

\begin{abstract}
Refractive errors are caused by a decoupling of the power of convergence of the eye lens, the cornea and lens, which make the rays reaching the eye to focus and generate an image, and the retina, which is the biological photosensitive screen where the image will be transformed into a nerve impulse. These defects include myopia, hyperopia and astigmatism. Presbyopia can also be considered a refractive defect, but of special features, since only affects near vision in patients older than 40 years. By altering the quality of the focused image on the most sensitive area of the retina (the macula), they decrease visual acuity. For their management several options exist, from the use of glasses and contact lenses to surgical correction (refractive surgery). The incidence of certain refractive errors (myopia specifically) has increased in recent decades, some environmental factors related to it have been identified. Some medical management measures have shown a positive effect in controlling its onset and progression.
\end{abstract}

Keywords: Refractive errors; presbyopia; myopia; hyperopia; astigmatism

1Centro Oftalmológico Virgilio Galvis. Floridablanca, Santander, Colombia.

2Departamento de Cirugía. Escuela de Medicina. Universidad Autónoma de Bucaramanga. Floridablanca, Santander, Colombia.

3Centro del Ojo, Guayaquil, Ecuador.

Correspondencia: Alejandro Tello, Centro Oftalmológico Virgilio Galvis. Centro Médico Ardila Lulle, Torre A, Piso 3, Mod. 7. Floridablanca,

Colombia. Correo electrónico: alejandrotello@gmail.com

Financiación: Este trabajo no recibió financiación de parte de ninguna entidad.

Los autores niegan tener algún conflicto de interés con respecto a este trabajo. 


\section{Introducción}

El fenómeno de la refracción de la luz permite en el ojo normal la generación de una imagen nítida en la retina. La alteración de este proceso genera los conocidos como defectos refractivos, por ser alteraciones en la refracción normal del ojo. También son llamadas ametropías, por que carecen de la condición normal del enfoque: emetropía.

Haremos una breve revisión histórica sobre ellos, y observaciones sobre sus características clínicas. El propósito es que el médico no oftalmólogo tenga claros los conceptos básicos sobre los defectos de refracción y su corrección óptica.

Historia de la corrección de los defectos de refracción

Desde la antigüedad (siglo IV a. C.) Aristóteles ya había realizado un análisis bastante certero del fenómeno de la visión, sugiriendo que los objetos alteraban un medio circundante y de esa manera eran observados por los ojos. (1) Sin embargo, solo hasta el siglo $X$ de nuestra era fue que Al-Hazen, físico considerado padre de la óptica moderna, afirmó que los rayos de luz reflejados en los objetos se dirigían hacia el ojo y de esa manera los objetos eran percibidos. El progreso subsiguiente de la comprensión de la óptica fisiológica fue muy lento y solo hasta 1604 fue cuando Kepler, basándose en los escritos de Al-Hazen, propuso la teoría de la imagen retiniana ${ }^{(1,2)}$.

En cuanto a ayudas ópticas, en la antigua Grecia se habían utilizado globos de vidrio llenos de agua como lentes para iniciar un fuego al condensar la luz solar ${ }^{(3)}$ pero la primera evidencia del empleo de ellos con el propósito de magnificar las imágenes y corregir la presbicia, proviene de Séneca en el siglo primero de nuestra era ${ }^{(1,3)}$.

Sin embargo, solo hasta mediados del siglo XIII fue cuando Roger Bacon construyó verdaderos lentes de vidrio (lupas) para la corrección de la presbicia, aunque no hay evidencia que en realidad fueran utilizados ${ }^{(3-5)}$. Bacon también mencionó lentes que podrían ser usados para ver objetos a gran distancia, adelantándose mas de tres siglos a lo que sería el telescopio que Galileo perfeccionaría ${ }^{(3)}$. Algunas décadas mas tarde, alrededor de 1285, aparecieron en Italia los primeros verdaderos anteojos, acoplados en un marco apoyado en el puente nasal y muy seguramente sostenidas con la mano, con lentes convergentes (positivos) para la corrección de la presbicia. Se han sugerido dos posibles nombres de su inventor: Alessandro da Spina o Salvino degli Armato, pero las evidencias son débiles ${ }^{(3-7)}$. El primer registro gráfico de estos anteojos se encuentra en un fresco de 1352 (Figura 1) ${ }^{(5-7)}$.

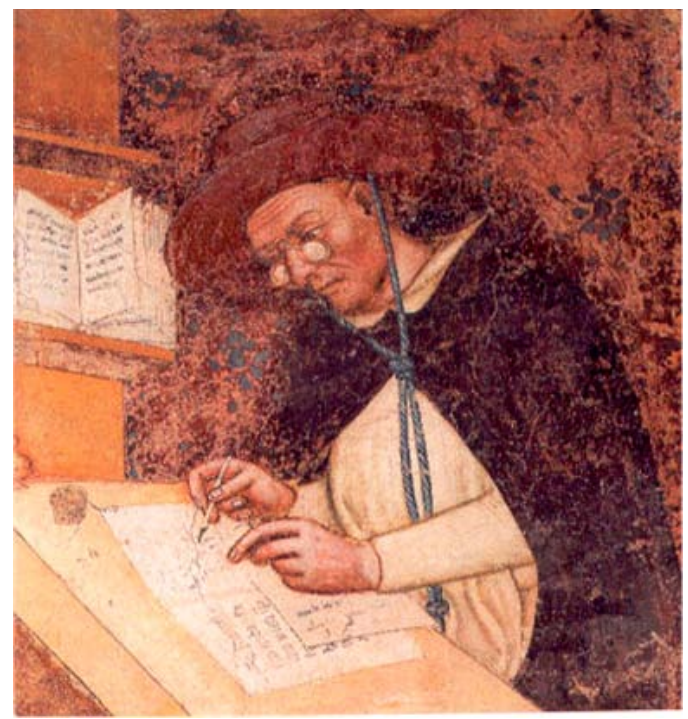

Figura 1: Primer registro gráfico que existe del uso de los anteojos en un fresco pintado por Tommaso da Modena en Treviso (Italia) en 1352, donde se muestra al Cardenal Hugo de Provenza con unos apoyados en el puente nasal. (Disponible en la página "http://www. college-optometrists.org/en/college/museyeum/online exhibitions/ spectacles/invention.cfm". Usado con permiso).

Nicolás de Cusa (1401-1464) hace la primera mención del uso de lentes negativos para la corrección de la miopía ${ }^{(8)}$. El uso de los anteojos se extendió lentamente durante los siglos XV a XVII. Sin embargo eran incómodos e inestables ${ }^{(9)}$. Se utilizaron algunos medios como cordeles o cintas, para intentar sostenerlos de manera mas estable, pero el avance mas importante se dio cuando Edward Scarlett en Londres, alrededor de 1730, les añadió unas extensiones laterales rectas que llegaban hasta detrás de las orejas terminando en espirales ${ }^{(5,10)}$. Estas extensiones laterales sufrieron posteriores diversas modificaciones: con anillos terminales, articuladas en la parte media de su extensión y finalmente con una curvatura terminal por detrás de las orejas, similar a como las conocemos actualmente, modificación desarrollada hacia mediados del siglo XIX (11).

Otro adelanto importante fue el invento de los 
bifocales, clásicamente atribuido a Benjamín Franklin aparentemente en 1.784, aunque existen dudas sobre ello ${ }^{(10,12)}$.

Una idea que fue sugerida inicialmente por Leonardo Da Vinci y luego también por trabajos de Descartes y Young, fue la de colocar el elemento de corrección óptica directamente sobre la córnea ${ }^{(13)}$. Este concepto se hizo realidad mucho después, con los lentes de contacto fabricados de vidrio, que fueron desarrollados inicialmente al final del siglo XIX. Mas de medio siglo mas tarde, a fines de la década de 1930, se empezaron a fabricar con materiales plásticos lo cual permitió que fuesen mucho mas precisos y además eran de mucho menor peso. Inicialmente se diseñaron con apoyo escleral y cubriendo la totalidad de la córnea. A principios de la década de 1950 se desarrollaron los lentes de contacto corneales, de menor diámetro, que funcionaron bastante bien y se diseminaron por todo el mundo. Unos años después se introdujo el material blando de hidrogel, y así nacieron los lentes de contacto blandos, de mejor tolerancia ${ }^{(14)}$. En 1987 fueron lanzados al mercado los lentes blandos desechables, para ser cambiados en periodos de entre un mes y tres meses, en material de hidrogel HEMA, con la ventaja teórica de menor riesgo de alergia e infecciones. Sin embargo diversos estudios de la época concluyeron que los eventos adversos seguían siendo muy similares a los presentados en los blandos convencionales, que se usaban en las horas de vigilia, hasta por un año ${ }^{(15,16)}$. Posteriormente estos riesgos fueron atribuidos mayormente al uso extendido (varias noches continuas de uso), siendo la queratitis infecciosa cinco veces más frecuente en el uso extendido que en el uso diario ${ }^{(17,18)}$. En 1990 se introdujeron los lentes blandos de hidrogel de silicona o HySi, con un incremento notorio en la transmisión de oxígeno $(19,20)$. Los lentes de contacto blandos modernos se fabrican con este material.

\section{Óptica fisiológica}

El ojo es equivalente a cualquier cámara que vemos conectada a un computador. Lo que hacen esas pequeñas cámaras es convertir las imágenes en impulsos eléctricos que llevan información digital, por el cable que las une, a la unidad interna del computador, donde está el procesador que interpreta esa información. Haciendo una analogía, el ojo es la cámara, el cable es el nervio óptico y el procesador es el cerebro. El ojo posee unos lentes convergentes (a los cuales por convención se les indica su poder en Dioptrías con signos positivos), que hacen que los rayos de luz se encuentren, formando un punto focal, el cual idealmente debe estar justo sobre la mácula (zona de la retina con mas densidad de fotorreceptores). El poder de convergencia sumado de los dos lentes (córnea y cristalino) es de aproximadamente + 64.00 Dioptrías, ya que el cristalino en reposo tiene aproximadamente +20.00 Dioptrías y la córnea posee en promedio un poder de +43.00 Dioptrías (Figura 2). Ahora, mientras que la córnea, es un lente estático, el cristalino tiene la capacidad de cambiar su forma e incrementar su poder óptico de convergencia ${ }^{(21,22)}$.

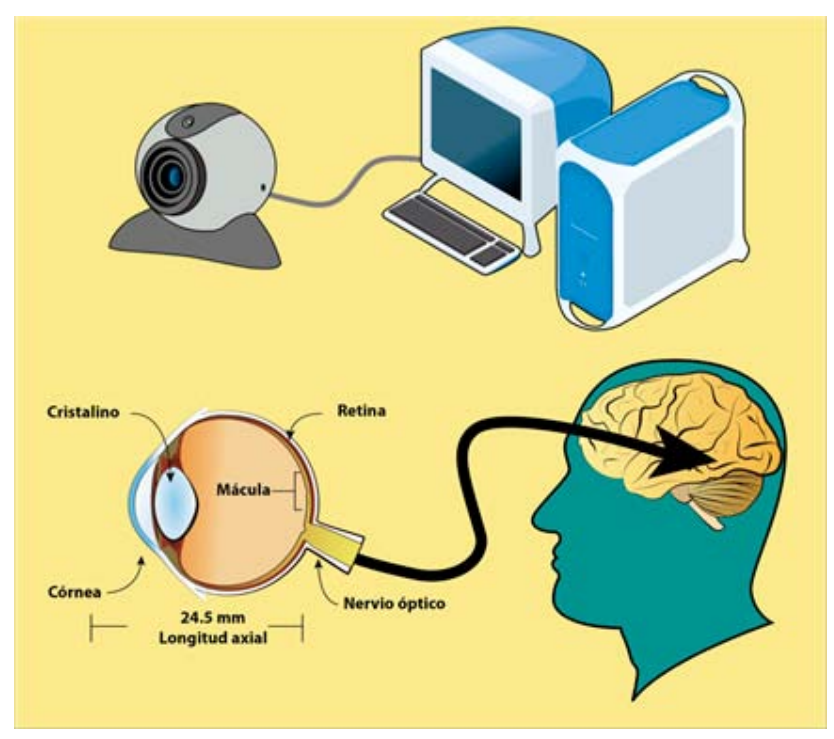

Figura 2: El ojo funciona como una cámara digital de un computador. Tiene unos lentes (córnea y cristalino) que enfocan la imagen en una pantalla sensible a la luz (retina) que la convierte en impulsos nerviosos que son transmitidos al cerebro por medio del nervio óptico.

Vemos los objetos por que estos emiten luz (objetos luminosos) o reflejan luz (objetos no luminosos). La luz visible sale de todos los objetos de manera omnidireccional, es decir en todas las direcciones (con excepción de los espejos que no generan reflexión difusa sino especular) y por ello en principio los rayos son de tipo divergente (21). Sin embargo cuando estos rayos han recorrido 
alrededor de 6 metros, la divergencia entre ellos es tan pequeña que se pueden considerar paralelos. Así, si un objeto se encuentra a menos de 6 metros del ojo, pero sobretodo si se encuentra a menos de un metro, los rayos de luz llegarán a la córnea siendo divergentes y entre menor sea la distancia, mas divergentes serán ${ }^{(21)}$. (Figura 3)

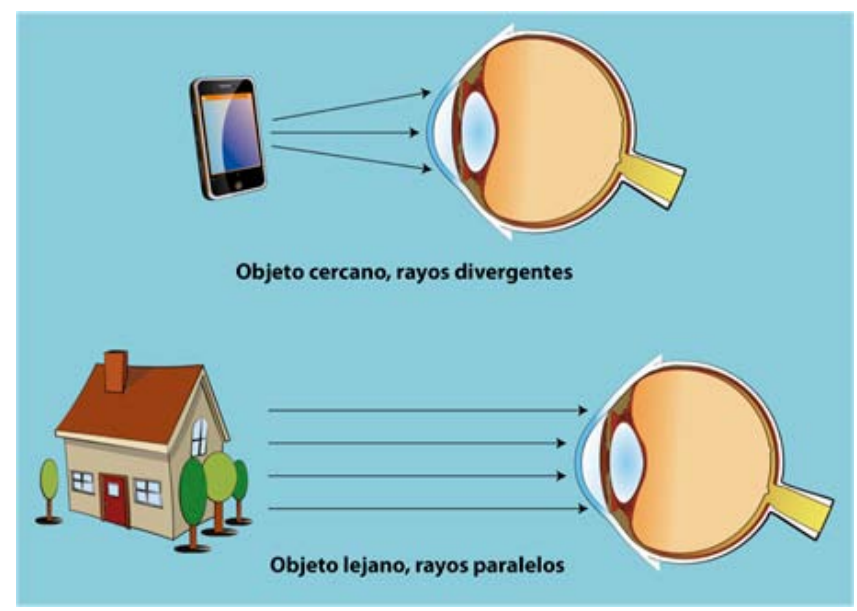

Figura 3: Los rayos de luz procedentes de un objeto cercano llegarán al ojo siendo divergentes, mientras que si provienen de un objeto lejano, llegarán siendo paralelos.

\section{Emetropía y ametropía}

Para determinar si un ojo tiene un defecto refractivo se usan como referencia los rayos paralelos procedentes de un objeto lejano, estando el ojo en reposo, es decir sin acomodación en el cristalino.

Un ojo emétrope es aquel en el cual, los rayos de luz paralelos se enfocan en la mácula, formando allí una imagen nítida. Sin embargo, la emetropía no equivale necesariamente a buena visión, ya que pueden existir pacientes emétropes, que tengan mala visión o inclusive que sean ciegos (por alteraciones de la mácula o del nervio) (Figura 4) ${ }^{(21)}$.

El ojo emétrope está así acoplado con un objeto lejano, pero si se enfrenta a un objeto cercano, al ser los rayos divergentes, el foco se desplazará hacia atrás, ubicándose en un punto posterior a la retina (Figura $5 \mathrm{~A}$ ). Como consecuencia, la imagen de ese objeto cercano formada en la mácula será borrosa. Para ver claramente los objetos cercanos es necesario entonces el aporte del

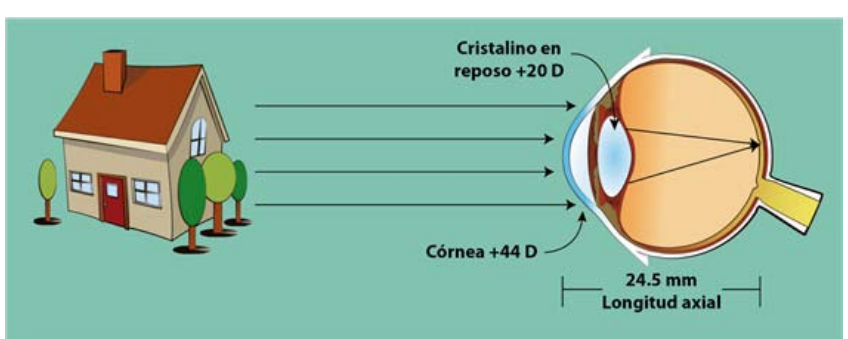

Figura 4: Emetropía. Los rayos paralelos, procedentes de un objeto lejano, se enfocan en la mácula.

cristalino mediante la acomodación. Este es un fenómeno que se caracteriza por la contracción del músculo ciliar, la relajación de las zónulas y el abombamiento del cristalino, lo que causa un aumento de sus curvaturas (especialmente de la cara anterior) y por ende de su poder de convergencia como lente positivo. Este fenómeno se desencadena de forma refleja cuando se enfoca una imagen por detrás de la retina, y como consecuencia desplazará el foco hacia adelante (Figura 5 B). La magnitud de la acomodación necesaria dependerá de la distancia a la que esté el objeto: si está mas cercano se requerirá mayor acomodación. A la distancia promedio de lectura de un texto (alrededor de $33 \mathrm{~cm}$ ), se deben acomodar aproximadamente $+3,00$ Dioptrías. Si se lee un monitor de un computador, cuya distancia es generalmente un poco mayor (entre 40 y 50 $\mathrm{cm})$, se requerirá acomodar entre $+2,50 y+2,75 \mathrm{D}$ (Figura $5 \mathrm{~B}$ ). Con el paso de los años el cristalino humano se endurece y se disminuye la amplitud de la acomodación. En general, después de los 40 años de edad la capacidad acomodativa cae por debajo de las tres dioptrías necesarias para una lectura cómoda y aparece la llamada presbicia. Los pacientes presentan dificultad para ver la letra pequeña y sienten la necesidad de alejar las cosas, ya que al ser mas lejano el objeto se requerirá menor esfuerzo acomodativo ${ }^{(21,22)}$.

Ahora, cuando no se cumple la condición ya definida de la emetropía, se presentan los defectos refractivos o ametropías. En ellas los rayos paralelos que llegan al ojo en reposo no se enfocan en la retina. Existen tres tipos: la miopía, la hipermetropía y el astigmatismo.

La miopía consiste en que los rayos de luz paralelos se enfocan en un punto anterior a la mácula. Esto se debe principalmente a que la longitud 


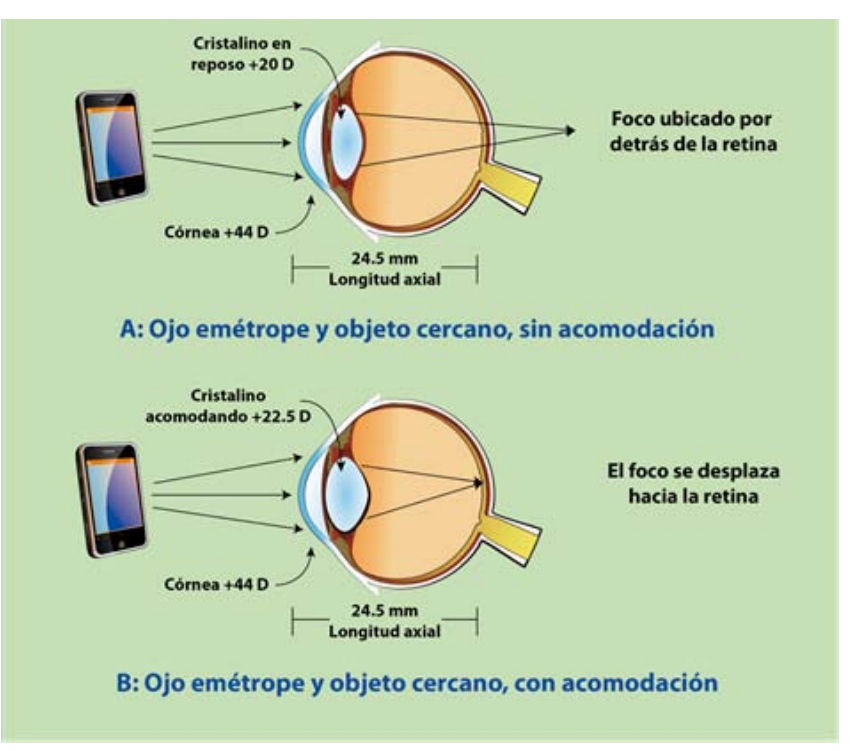

Figura 5: A: Al enfrentarse a los rayos divergentes de un objeto cercano, en un ojo emétrope se formará la imagen por detrás de la retina. B: Para poder ver con claridad se necesita que el ojo incremente su poder óptico, acomodando, y de esa manera se desplace el foco hacia adelante.

axial del ojo es excesiva; otra razón es el aumento en el poder de la córnea. Generalmente se presenta una combinación de estas condiciones (21,23). En otros casos la miopía puede generarse por cambios en el poder del cristalino, como en casos de catarata nuclear incipiente. La miopía alta, es decir mayor de 6.00 Dioptrías se puede acompañar de alteraciones importantes en la retina, con riesgo de desprendimiento de retina ${ }^{(24)}$. El paciente miope verá borrosos los objetos lejanos (Figura 6 A). Por otra parte, como los rayos de luz procedentes de los objetos cercanos llegan al ojo siendo divergentes, su punto de enfoque se desplazará hacia atrás, y en el ojo miope alcanzará a la retina. Por esta razón los miopes tienen buena visión cercana sin necesidad de ejercer acomodación. Como una consecuencia de este fenómeno, cuando los miopes son mayores de 40 años y tienen presbicia, pueden seguir leyendo de cerca sin usar gafas. No es por que no sufran de presbicia ( pues de hecho la tienen y no pueden acomodar) sino por que ópticamente no necesitan acomodar para ver claro estos objetos cercanos (Figura 6 B). La miopía se corrige usando lentes divergentes (negativos). De esa manera los rayos paralelos se convierten en divergentes antes de atravesar la córnea, y se desplaza hacia atrás el punto focal, llegando a la retina.

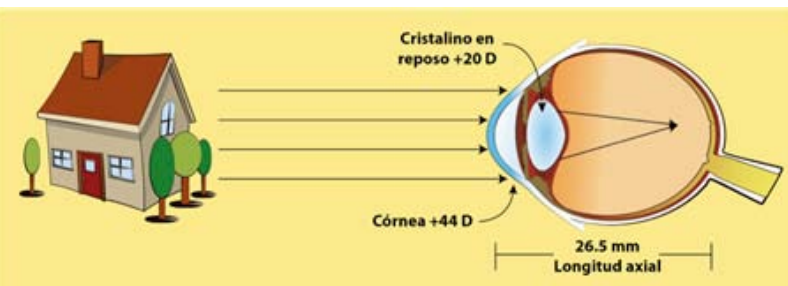

A: Ojo miope y objeto lejano, foco delante de la retina

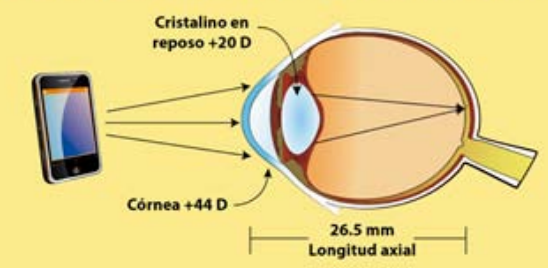

B: Ojo miope y objeto cercano, foco se desplaza a la retina

Figura 6: Miopía. A: Los rayos paralelos se enfocan antes de la retina, y la visión lejana es borrosa. B: Los rayos divergentes de un objeto cercano se enfocan mas posteriormente, llegando a la mácula y por tanto se ven de forma clara, sin necesidad de ejercer acomodación.

En la hipermetropía la longitud axial del ojo, al contrario que en la miopía, usualmente es muy pequeña, o la córnea tiene menor poder dióptrico del necesario, o, como ocurre con frecuencia, se encuentra una combinación de estas dos condiciones ${ }^{(21,23)}$. Ello causa que los rayos paralelos provenientes de los objetos lejanos se enfoquen por detrás de la retina (Figura $7 \mathrm{~A}$ ). Cuando el paciente hipermétrope es muy joven, la presencia de esa imagen enfocada en un punto posterior a la retina desencadenará el reflejo de la acomodación, haciendo que se incremente el poder dióptrico del cristalino, y el paciente así puede ver claro los objetos lejanos (Figura 7 B). Inclusive los niños hipermétropes, como por su edad tienen amplitudes de acomodación de hasta +15.00 Dioptrías, pueden ver claro también objetos cercanos ya que pueden acomodar sin problema las aproximadamente $+3,00$ Dioptrías adicionales necesarias. Sin embargo, con el incremento de la edad del paciente, la visión próxima se hace cada vez mas difícil, y usualmente en la adolescencia o la adultez temprana empiezan a presentarse síntomas de cansancio al leer y desenfoque de las letras impresas (astenopia). Ahora, cuando el hiper- 
métrope llega a la presbicia, no puede compensar el defecto y por ello, además de ver borroso de cerca también verá desenfocados los objetos lejanos. La hipermetropía se corrige usando lentes convergentes (positivos).

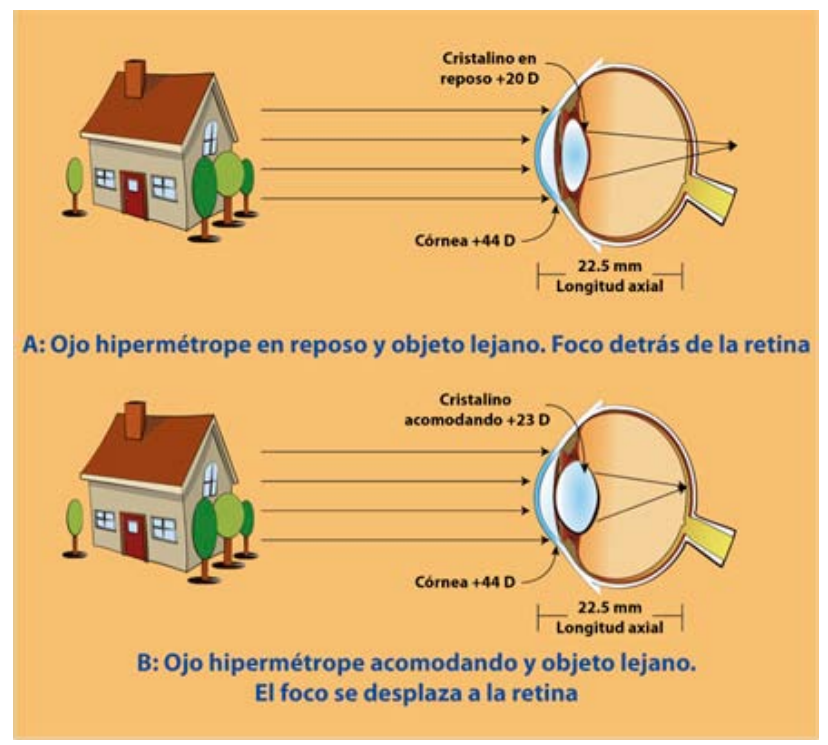

Figura 7: Hipermetropía. A: Los rayos paralelos procedentes de un objeto lejano se enfocan por detrás de la retina. B: Si el ojo tiene una hipermetropía de $+3.00 \mathrm{D}$, al acomodar esa magnitud, como lo hace el ojo de una persona joven, podrá enfocar el objeto lejano adecuadamente.

El astigmatismo se relaciona con diferencias de poder entre los diferentes meridianos del sistema óptico del ojo. Aunque puede ser generado tanto por la córnea como por el cristalino, en general el determinante de los astigmatismos significativos clínicamente es la córnea. Para explicarlo de una manera sencilla, una córnea sin astigmatismo es similar a la mitad de una esfera, esto es como si tomáramos un balón de basquetbol y lo cortáramos en dos. El meridiano vertical tendrá una curvatura igual a la del meridiano horizontal, y a la de todos los meridianos intermedios. La córnea con astigmatismo, por el contrario, se asemeja a un balón de futbol americano cortado a la mitad. El meridiano horizontal será menos curvo ( $y$ tendrá por lo tanto menor poder dióptrico) que el meridiano vertical. Entre estos dos meridianos ortogonales (llamados así por estar a 90 grados de distancia) existen un número infinito de meridianos intermedios con un poder que irá siendo creciente desde la posición horizontal hasta la verti- cal (Figura 8). Ahora, aunque la diferencia entre los poderes de los diversos meridianos determina el astigmatismo, el tipo exacto de esta ametropía depende de la relación de los dos focos extremos, con respecto a la retina, lo cual está determinado por la longitud axial del ojo.
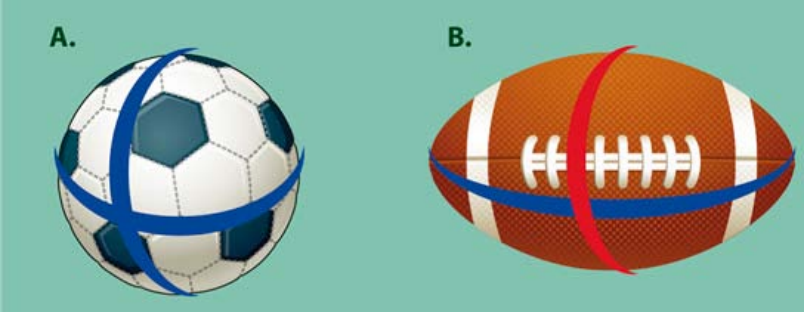

Figura 8. A: En una córnea sin astigmatismo todos meridianos tiene similar curvatura. Se comporta como media esfera (como un balón de basquetbol). B: La córnea con astigmatismo tiende a ser ovalada, como un balón de futbol americano, con un meridiano más curvo (rojo) y uno más plano (azul). Todos los meridianos intermedios tendrán un poder variable: en este ejemplo creciente desde la posición horizontal hasta la vertical.

De acuerdo a ello el astigmatismo se clasifica en: miópico simple, miópico compuesto, mixto, hipermetrópico simple o hipermetrópico compuesto (Figura 9).

Para su corrección óptica los astigmatismos requieren el uso de lentes esfero-cilíndricos, es decir que tienen diferentes poderes en diversos meridianos, con el fin de compensar las diferencias de poder del sistema óptico del ojo.

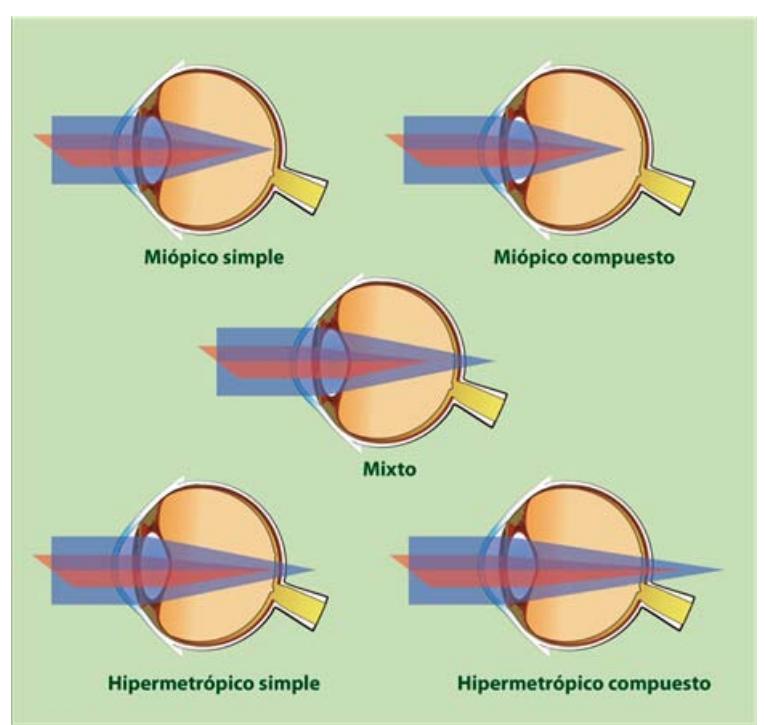

Figura 9. Tipos de astigmatismo de acuerdo a la relación de los dos focos extremos, con la retina.

Etiología y epidemiologia de los defectos refractivos 
Tanto factores hereditarios como ambientales juegan un papel importante en el desarrollo de los defectos refractivos ${ }^{(25-27)}$. Sin embargo, los genes identificados hasta ahora explican solo un pequeño porcentaje de las ametropías ${ }^{(27)}$. Por otra parte, cada vez hay mas evidencia de la influencia de los factores ambientales en la determinación de ciertos defectos refractivos, y específicamente en la miopía, al parecer con mas efecto en la edad escolar ${ }^{(28-32)}$. El notable incremento de la prevalencia de miopía en el transcurso de solo dos generaciones en ciertas áreas del mundo (Hong Kong, Singapur, Taiwán, China y Corea del Sur), que está constituyendo una verdadera epidemia en esos países, no puede explicarse solo por factores genéticos ${ }^{(33-35)}$. Las prevalencias recientemente encontradas son alarmantes: en China se reportó en 2012 una tasa de miopía de un 94,9\% en universitarios (promedio de edad 18,8 años) y entre los jóvenes coreanos de 19 años se encontró una prevalencia del $96,5 \%$ en $2010{ }^{(34,35)}$.

El trabajo en visión próxima se ha asociado con la progresión de la miopía, mientras que las actividades al aire libre (relacionadas con la exposición de los ojos a la luz solar) parecerían proteger contra la progresión de ella ${ }^{(28,29,36,37)}$. Por esto, el recomendar disminuir el sobreuso de las actividades de visión próxima (lectura, computadores, tabletas electrónicas, teléfonos celulares inteligentes) e incrementar la exposición a la luz solar, aún con el uso de bloqueadores UV en la piel y sombreros, podrían ser factores que ayuden al control de la aparición y progresión de la miopía ${ }^{(28,38)}$.

\section{Historia natural de las ametropías}

Los recién nacidos tienen en promedio una hipermetropía de 3 Dioptrías, pero esta disminuye rápidamente y menos del $5 \%$ de los niños tienen ese defecto a la edad de un año ${ }^{(39)}$. El ojo normalmente crece de manera significativa hasta los 6 años de edad, y luego prácticamente se detiene su elongación.

La miopía típicamente aparece ente los 6 y 12 años de edad y su rata de progresión, si no se hace ninguna intervención terapéutica, es aproximadamente de 0.30 a 0.50 Dioptrías por año, relacionándose con un crecimiento anormalmente persistente del globo ocular, que no se detiene sino hasta el final de la adolescencia o la adultez temprana (18 a 21 años), aunque en un porcentaje de casos (del $17,2 \%$ en un estudio a largo plazo en Finlandia) puede progresar mas de 1,00 Dioptría por encima de los 24 años de edad (40). En algunos grupos poblacionales asiáticos se ha encontrado que la miopía puede aparecer mucho mas tempranamente (alrededor de los 3 años de edad) ${ }^{(25)}$.

En los hipermétropes aunque su defecto en realidad no progresa (el ojo no se "encoje"), si se manifiesta cada vez más con el paso del tiempo, ya que progresivamente deja de ser compensado por la acomodación. Esto quiere decir que niños y adolecentes con hipermetropía pueden lograr buena visión aun sin usar ningún tipo de corrección óptica; pero hacia la tercera década de la vida van notando primero problemas de visión próxima, y después de los 40 años al iniciarse la presbicia, se incrementa esta limitación y aparecen también dificultades con la visión lejana, pues ya no pueden compensar su defecto con la acomodación (21).

El astigmatismo cambia poco durante la vida en su magnitud, pero si en su orientación. En niños y jóvenes es comúnmente "con la regla" es decir con el meridiano más plano a 180 grados. En los adultos mayores el astigmatismo tiende a cambiar y volverse "contra la regla", con el meridiano más plano a 90 grados ${ }^{(41)}$.

\section{Riesgos asociados con las ametropías}

Los individuos con errores refractivos altos tienen más riesgo de desarrollar otras patologías oculares. En miopes altos los mayores riesgos se relacionan con los problemas degenerativos de la retina ya que al ser el globo de un tamaño desproporcionadamente largo, la retina se "estira" y presenta áreas de atrofia en la mácula que puede afectar la visión, o adelgazamientos y rupturas en la periferia, que pueden llevar a desprendimiento de la retina. Adicionalmente tienen un mayor riesgo de presentar glaucoma crónico de ángulo abierto ${ }^{(42,24) .}$

Los hipermétropes, por su parte, al tener el ojo mas pequeño disponen de menor espacio dentro del globo para las estructuras anatómicas y por ello tienen mayor riesgo de presentar glaucoma de ángulo estrecho. Este riesgo se presenta por 
encima de los 40 años, debido a que el cristalino crece durante toda la vida, y después de esa edad, por su tamaño, tiende a empujar al iris hacia adelante y a estrechar el ángulo irido-corneal ${ }^{(43)}$. Por estos motivos, tanto los miopes como los hipermétropes altos deben ser evaluados al menos cada 2 años por el especialista.

\section{Corrección con gafas y lentes de contacto}

Prescribir una corrección óptica en mayores de 12 años, depende de dos factores; el primero es la sintomatología, lo cual quiere decir que en defectos refractivos que no generen ningún tipo de molestia (por lo general ametropías bajas) no se justifica adaptar ningún tipo de corrección, pero si existe un componente sintomatológico, sobre todo relacionado con actividades de fijación prolongada (lectura, escritura, uso del computador, televisión) es mandatorio adaptar la corrección. El segundo factor es el entorno del paciente, las exigencias visuales y el grado de afección de la agudeza visual; esto quiere decir que el mismo paciente es quien reconoce si tiene o no algún grado de limitación de acuerdo a su propio entorno. De esta forma, habrá pacientes adultos que aún teniendo un defecto refractivo con disminución de la agudeza visual podrán optar por no utilizar ningún tipo de corrección óptica siempre y cuando no se vean afectadas sus necesidades sin generarse ningún efecto contraproducente. Por otra parte, en la primera década de la vida es obligatorio utilizar la corrección óptica en los defectos clínicamente significativos, ya que existe riesgo de ambliopía (mal desarrollo de la vía visual por una mala estimulación) ${ }^{(44)}$.

De igual manera en la miopía juvenil también es necesario utilizar la corrección óptica, pues la evidencia científica sugiere que la ausencia de corrección o la hipo-corrección favorecen a la progresión de la miopía ${ }^{(45-47)}$.

La corrección óptica con gafas suele ser la alternativa de primera elección por ser la menos invasiva, la más sencilla y la más económica.

Cuando un ojo es emétrope, no requiere ningún aumento para poder enfocar los rayos paralelos. Por ello decimos que su defecto refractivo es cero. En la literatura científica en español esto se denota con una "N", por "Neutro" ( en inglés se colocan las letras "PI" por la palabra "Plano"). Los defectos de miopía y astigmatismo se corrigen ópticamente con gafas que tienen lentes esféricos, divergentes (negativos) para la primera y convergentes (positivos) para la segunda. Una prescripción óptica para un paciente miope será por ejemplo: $-3.00 \mathrm{D}$. Esto quiere decir que con un lente divergente de 3.00 Dioptrías colocado en las gafas, los rayos paralelos se separarán justo lo suficiente para desplazar el foco hacia atrás para que llegue a la mácula. Como el lente tiene el mismo poder en todos los meridianos, lo llamamos lente esférico, y como el defecto refractivo se corrige con este tipo de lente, también se denomina defecto esférico. En el caso de un paciente hipermétrope sería por ejemplo: +3.00 D.

Para corregir astigmatismos es mas complejo, se requiere un lente que tiene una combinación de una esfera y un cilindro (esfero-cilíndrico), que tiene diferentes poderes en diferentes meridianos. Estas prescripciones tienen además un eje, para poder colocar el lente en la posición adecuada para ese ojo en particular. Un ejemplo de una corrección para astigmatismo sería: $-1.00-1.00 \mathrm{x}$ $180^{\circ}$. El primer número se conoce como la "esfera", el segundo como el "cilindro" y el tercero como el "eje".

La corrección óptica de la presbicia es un caso especial, pues como se presenta universalmente, los ojos pueden tener cualquier estado refractivo de base. Es decir, en un emétrope, quien no requiere ninguna corrección para la visión lejana, se requerirá colocarle un poder positivo solo para cuando vaya a usar la visión cercana (para reemplazar el incremento de la convergencia óptica que el cristalino ya no puede generar). La magnitud del poder de este lente positivo (denominado en las fórmulas ópticas "adición" o "Add") se relaciona estrechamente con la edad: un paciente de 40 años requerirá aproximadamente $+1.00 \mathrm{D}$ de adición, mientras que un paciente de 60 años, que prácticamente no ejerce ya ninguna acomodación con el cristalino, requerirá una adición de aproximadamente $+3.00 \mathrm{D}$.

Ahora, cuando el paciente usa la adición ve bien de cerca, pero si la usa para visión lejana, el sistema óptico tendrá demasiado poder y el foco se desplazará delate de la retina (es decir el ojo se 
miopizará) y el paciente verá borroso.

Por ello es que se prefiere en estos casos los lentes de tipo bifocal, que tienen un diferente poder óptico en la parte superior y que en la parte inferior. La nueva versión de este tipo de lentes son los multifocales o progresivos, que no tienen solo dos aumentos, sino muchos, y por ello permiten el enfoque a diversas distancias (cercana para la lectura, intermedia para el computador y lejana). Cuando el ojo presenta otro tipo de defecto refractivo, se le añade esta corrección a las gafas, de manera que el paciente ve bien con ellas a cualquier distancia.

Por ejemplo en un emétrope présbita de 65 años, la fórmula sería:

N. Add: $+3,00 \mathrm{D}$.

En un miope de 65 años de edad:

-3.00 D. Add: +3.00 D

En el lente correspondiente a la lectura, la adición se adiciona a la corrección de lejos, y por ello en el caso del miope presentado, en realidad la zona de lectura cercana no tiene ningún aumento $(-3.00+3.00=0)$, por que el miope de esa magnitud puede leer sin necesidad de acomodar, según lo ya explicado anteriormente.

En un hipermétrope de 65 años:

+3,00 D Add: +3,00 D.

En este caso la zona del lente dedicada a la visión de lectura tendrá en total un poder óptico de $+6,00 \mathrm{D}$.

La otra opción para la corrección óptica de los defectos refractivos son los lentes de contacto, que se colocan directamente sobre la córnea. Los lentes de contacto pueden adaptarse en cualquier edad y en cualquier tipo de defecto refractivo; pero siempre con la adecuada educación sobre su uso. Es fundamental aprender a utilizar las soluciones de limpieza y mantenimiento, y limitar las horas de uso del lente de contacto solo durante el día (idealmente un máximo de 10 horas), nunca dormir con los lentes de contacto puestos, alternar con anteojos y descansar un día a la semana de los lentes de contacto. En casi todos los casos se pueden emplear lentes de contacto blandos (fabricados en hidrogel de silicona), ya que si hay un astigmatismo alto se les puede colocar corrección cilíndrica (los llamados lentes "tóricos"). Sin embargo en casos de defectos astigmáticos muy altos o irregulares, se pueden requerir lentes rígidos de un plástico especial permeable al gas. Como ya se mencionó, el sobreuso de los lentes de contacto, y el usarlos durante el sueño, incrementa en cinco veces el riesgo de una infección corneal ${ }^{(16-18)}$.

\section{Ortoqueratología}

Consiste en el aplanamiento "temporal" de la córnea central para corregir la miopía, mediante el uso de lentes de contacto rígidos diseñados especialmente solo para el uso nocturno (mientras el usuario duerme) y para retirarlos durante el día. De esta manera se espera que la córnea mantenga la forma mas aplanada durante las horas del día, y el paciente pueda tener buena visión sin necesidad de ninguna ayuda óptica, durante ese tiempo. En los últimos 10 años se ha incrementado su uso (sobretodo en las países Asiáticos, pero también en Europa y el continente americano), y se ha encontrado que además de permitir la corrección óptica transitoria de la miopía, ayuda a detener la progresión del defecto (es decir tienen un efecto dual) reduciéndola hasta en $45 \%{ }^{(48,49)}$.

El caso especial de la miopía : alternativas para su prevención y control

Mientras que la hipermetropía es un defecto que se manifiesta normalmente desde la primera infancia, presenta una disminución con el proceso normal de crecimiento del ojo y se estabiliza alrededor de la pubertad; la miopía por lo contrario, tiende a progresar desde la infancia hasta la segunda o tercera década de la vida, pudiendo llegar a niveles muy altos ${ }^{(40)}$.

Múltiples alternativas se han empleado para tratar de controlar dicha progresión y la que ha demostrado mayor efectividad es el uso tópico del anticolinérgico atropina ${ }^{(50,51)}$. Nosotros hemos tenido experiencia positiva con el uso de atropina al $1 \%$ una vez a la semana ${ }^{(52)}$. Adicionalmente se ha demostrado que aumentar las horas al día de exposición al aire libre tiene un efecto protector ${ }^{53-}$ 55). Incrementar los niveles de luz para realizar tares de visión próxima, han sido también sugeridos como factores protectores en cuanto a la progresión de la miopía ${ }^{(56,57)}$. Como se han determinado que los periodos continuos de mas de 45 minutos de trabajo en visión próxima, también constituyen 
un riesgo de progresión, se recomienda realizar pausas en la lectura, y evitar actividades de visión próxima no indispensables ${ }^{(36,58)}$.

\section{Cirugía refractiva}

Son los procedimientos quirúrgicos que cambian el poder del sistema óptico del ojo para corregir una ametropía. Se pueden realizar por medio de la aplicación de un láser para modificar la córnea, con la implantación de un lente negativo o positivo en un ojo con cristalino (lentes intraoculares fáquicos) o extrayendo el cristalino y reemplazándolo por otro lente intraocular artificial del poder adecuado ${ }^{(59-61)}$.

\section{Conclusión}

Los defectos refractivos (ametropías) son de presentación frecuente. Actualmente las opciones de corrección con gafas o lentes de contacto son en general muy exitosas. También existen las alternativas quirúrgicas (cirugía refractiva). En el caso de la miopía, algunas recomendaciones de cambios en las actividades de niños y adolescentes pueden ayudar a frenar su progresión.

\section{Agradecimientos}

A Sandra Milena Carmona por su diligente ayuda en la revisión del texto del manuscrito, confirmando que estuviese en un lenguaje entendible para personas no expertas en oftalmología, así como por su cuidadoso trabajo en la organización de las referencias citadas.

\section{Conceptos clave}

-En el ojo emétrope los rayos procedentes de un objeto lejano se enfocan la mácula, en un ojo miope antes de ella y en un hipermétrope por detrás de ella.

- El astigmatismo se debe a diferencias en los poderes de los meridianos del sistema óptico, principalmente de la córnea.

-La presbicia es la pérdida de la flexibilidad del cristalino relacionada con la edad, que conlleva la pérdida de la acomodación.

-Los lentes esféricos se usan para corregir la miopía (lentes divergentes o negativos) y la hipermetropía (lentes convergentes o positivos). -Los lentes esfero-cilíndricos, que tienen diferentes poderes a lo largo de sus meridianos, se usan para corregir el astigmatismo.
-Se han identificado factores ambientales que influyen en la aparición y la progresión de la miopía, tales como el sobreuso de la visión próxima y la menor exposición a la luz solar.

\section{Referencias}

1. Findlen $P$, Bence $R$. The history of the eye. En: $\quad$ https://web.stanford.edu/class/history13/earlysciencelab/body/eyespages/eye.html; consultado el 1/10/2016.

2. Crombie AC. Expectation, modelling and assent in the history of optics: Part I. Alhazen and the Medieval tradition. Stud Hist Phil Sci. 1991;21:605-32. 3. King $\mathrm{H}$. Early references to lenses and to optics. In: The history of telescope. Mineola, Estados Unidos: Dover Publications Ins.; 1955. p. 27-9.

4. James $R$. The father of British optics: Roger Bacon, c. 1214-1294. Br J Ophthalmol. 1928;12:1-14. 5. Handley $N$. The invention of spectacles En: http://www. college-optometrists.org/en/college/museyeum/online_exhibitions/spectacles/invention.cfm; consultado el $\overline{1 / 10 / 2016 . ~}$

6. Ilardi V. The invention of Spectacles revisited. In: Renaissance Vision from Spectacles to Telescopes. Filadelfia, Estados Unidos: American Philosophical Society; 2007. p. 3-50.

7. Norman J. The Earliest Depiction of Eyeglasses in a Painted Work of Art (1352). En: http://www. historyofinformation.com/expanded.php?id=1755; consultado el 1/10/2016.

8. Goes F. Invention of Spectacles. In: The Eye in History. Nueva Deli, India: Jaypee Brothers Medical Publishers Ltd.; 2013. p. 126-33.

9. Handley N. Seventeenth century spectacles. En: http://www.college-optometrists.org/en/college/ museyeum/online_exhibitions/spectacles/seventeen. cfm; consultado el 1/10/2016.

10. Fleishman DA. Eyeglasses Through the Ages. En: http://www.antiquespectacles.com/history/ages/ through_the_ages.htm; consultado el 1/10/2016.

11. Handley N. A bit on the side - The development of spectacle sides. En: http://www.college-optometrists.org/en/college/museyeum/online_exhibitions/ spectacles/side.cfm; consultado el 1/10/2016.

12. Handley N. The "inventor" of bifocals? En: http://www.college-optometrists.org/en/college/museyeum/online_exhibitions/artgallery/bifocals.cfm; consultado el 2/10/2016.

13. Young T. On the mechanism of the eye. Philos Trans $R$ Soc London. 1801;91:23-88.

14. Sánchez Ferreiro A, Muñoz Bellido L. Evolución histórica de las lentes de contacto. Arch Soc Esp 
Oftalmol. 2012;87:265-6.

15. Boswall G, Ehlers W, Luistro A, Worrall M, DonshikPC. A comparison of conventional and disposable extended wear contact lenses. CLAO J. 1993;19:15865.

16. Poggio E, Abelson M. Complications and symptoms in disposable extended wear lenses compared with conventional soft daily wear and soft extended wear lenses. CLAO J. 1993;19:31-9.

17. Schein O, Glynn R, Poggio E, Seddon J, Kenyon $K$. The relative risk of ulcerative keratitis among users of daily wear and extended-wear soft contact lenses: a case control study. N Engl J Med. 1989;321:7738.

18. Poggio E, Glynn R, Schein O. The incidence of ulcerative keratitis among users of daily-wear and extended-wear soft contact lenses. N Engl J Med. 1989;321:779-83.

19. Morgan P, Efron N, Hill E, Raynor M, Whiting M, Tullo A. Incidence of keratitis of varying severity among contact lens wearers. Br J Ophthalmol. 2005;89:430-6.

20. Barr J. History and development of contact lenses. In: Bennett E, Weissman B, editors. Clinical contact lens practice. Segunda ed. Filadelfia, Estados Unidos: JB Lippincott Co; 2005. p. 1-10.

21. Katz M, Kruger P. The Human Eye as an Optical System. In: Tasman W, Jaeger EA, editors. Duane 's Ophthalmology. Filadelfia, Estados Unidos: Lippincott Williams \& Wilkins; 2013.

22. Galvis V, Tello A, Carreño N. El cristalino para el médico general. Med UNAB. 2008;11:225-30.

23. Barcsay G, Nagy Z, Németh J. Distribution of axial, corneal, and combined ametropia in a refractive surgery unit. Eur J Ophthalmol. 2003;13:739-44.

24. Saw SM, Gazzard G, Shin-Yen EC, Chua WH. Myopia and associated pathological complications. Ophthalmic Physiol Opt. 2005;25:381-91.

25. Chua SYL, Ikram MK, Tan CS, Lee YS, Ni Y, Shirong $C$, et al. Relative Contribution of Risk Factors for Early-Onset Myopia in Young Asian Children. Investig Opthalmology Vis Sci [Internet]. 2015;56:8101. Available from: http://iovs.arvojournals.org/article. aspx?doi=10.1167/iovs. 15-16577

26. Peet JA, Cotch MF, Wojciechowski R, BaileyWilson JE, Stambolian D. Heritability and familial aggregation of refractive error in the Old Order Amish. Investig Ophthalmol Vis Sci. 2007;48:4002-6.

27. Tkatchenko A V., Tkatchenko T V., Guggenheim JA, Verhoeven VJM, Hysi PG, Wojciechowski $R$, et al. APLP2 Regulates Refractive Error and Myopia Development in Mice and Humans. PLoS Genet. 2015;11:1-25.

28. Ramamurthy D, Lin Chua S, Saw S. A review of environmental risk factors for myopia during early life, childhood and adolescence. Clin Exp Optom. 2015;98:497-506.

29. French A, Ashby R, Morgan I, Rose K. Time outdoors and the prevention of myopia. Exp Eye Res. 2013;114:68.

30. Morgan IG, Ohno -Matsui K SS. Myopia. Lancet. 2012;379:1739-48.

31. Lougheed T. Myopia: the evidence for environmental factors. Env Heal Perspect. 2014;122:A12-9.

32. Wu P-C, Tsai C-L, Hu C-H, Yang Y-H. Effects of outdoor activities on myopia among rural school children in Taiwan. Ophthalmic Epidemiol. 2010;17:33842.

33. Lin LLK, Shih YF, Hsiao CK, Chen CJ. Prevalence of Myopia in Taiwanese Schoolchildren: 1983 to 2000. Ann Acad Med Singapore. 2004;33:27-33.

34. Jung SK, Lee JH, Kakizaki H, Jee D. Prevalence of myopia and its association with body stature and educational level in 19-year-old male conscripts in Seoul, South Korea. Investig Ophthalmol Vis Sci. 2012;53:5579-83.

35. Sun J, Zhou J, Zhao P, Lian J, Zhu H, Zhou Y, et al. High prevalence of myopia and high myopia in 5060 Chinese University students in Shanghai. Investig Ophthalmol Vis Sci. 2012;53:7504-9.

36. Huang $H$, Chang $D, W u$ P. The Association between Near Work Activities and Myopia in ChildrenA Systematic Review and Meta-Analysis. PLoS One. 2015;10:e0140419.

37. Jones $L A$, Sinnott $L T$, Mutti $D O$, Mitchell $G L$, Moeschberger ML, Zadnik K. Parental history of myopia, sports and outdoor activities, and future myopia. Investig Ophthalmol Vis Sci. 2007;48:3524-32.

38. Morgan IG. What Public Policies Should Be Developed to. Optom Vis Sci. 2016;93:1058-60.

39. Saunders KJ. Early refractive development in humans. Surv Ophthalmol. 1995;40:207-16.

40. Parssinen O, Kauppinen M, Viljanen A. The progression of myopia from its onset at age $8-12$ to adulthood and the influence of heredity and external factors on myopic progression. A 23-year follow-up study. Acta Ophthalmol. 2014;92:730-9.

41. Topuz H, Ozdemir M, Cinal A, Gumusalan Y. Age-related differences in normal corneal topography. Ophthalmic Surg Lasers Imaging. 2004;35:298-303.

42. The Eye Disease Case-Control Study Group . Risk factors for idiopathic rhegmatogenous retinal detachment. Am J Epidemiol. 1993;137:749-57.

43. Lowe R. Causes of shallow anterior chamber in primary angle-closure glaucoma. Am J Ophthalmol. 1969;67:87-93.

44. Tailor V, Bossi M, Greenwood J, Dahlmann- 
Noor A. Childhood amblyopia: current management and new trends. Br Med Bull. 2016;119:75-86.

45. Adler D, Millodot M. The possible effect of undercorrection on myopic progression in children. Clin Exp Optom. 2006;89:315-21.

46. Vasudevan B, Esposito C, Peterson C, Coronado $C$, Ciuffreda KJ. Under-correction of human myopia - Is it myopigenic?: A retrospective analysis of clinical refraction data. J Optom. Spanish General Council of Optometry; 2014;7:147-52.

47. Galvis V, Tello A, Blanco O, Parra MM. Refractive correction and myopia progression. Graefe's Arch Clin Exp Ophthalmol. 2016;254:407-8.

48. Koffler BH, Sears JJ. Myopia control in children through refractive therapy gas permeable contact lenses: Is it for real? Am J Ophthalmol. 2013;156:10761081.e1.

49. Hiraoka T, Kakita T, Okamoto F, Takahashi $H$, Oshika T. Long-term effect of overnight orthokeratology on axial length elongation in childhood myopia: a 5-year follow-up study. Invest Ophthalmol Vis Sci. 2012;53:3913-9.

50. Chia A, Lu Q, Tan D. Five-Year Clinical Trial on Atropine for the Treatment of Myopia 2: Myopia Control with Atropine $0.01 \%$ Eyedrops. Ophthalmology. 2016;123:391-9.

51. Galvis V, Tello A, Parra MM, Rodriguez CJ, Blanco O. Re: Chia et al.: Five-year clinical trial on atropine for the treatment of myopia 2: myopia control with atropine $0.01 \%$ eyedrops (Ophthalmology 2016;123:391-9). Ophthalmology. 2016;123:e40-1.

52. Galvis V, Tello A, Rodriguez CJ, Rey JJ. Atropine dose to treat myopia. Ophthalmology. 2012;119:1718.

53. Rose KA, Morgan IG, Ip J, Kifley A, Huynh S, Smith $W$ et al. Outdoor activity reduces the prevalence of myopia in children. Ophthalmology. 2008;115:127985.
54. Wu PC, Tsai CL, Wu HL, Yang YH, Kuo HK. Outdoor activity during class recess reduces myopia onset and progression in school children. Ophthalmology. 2013;120:1080-5.

55. Galvis V, Tello A, Castellanos $Y$, Camacho $P$, Prada A, Rangel C. Re: Wu et al.: Outdoor activity during class recess reduces myopia onset and progression in school children (Ophthalmology 2013;120:10801085). Ophthalmology. 2014;121:e20.

56. Hua $W J$, Jin $J X, W u X Y$, Yang JW, Jiang $X$, Gao GP, et al. Elevated light levels in schools have a protective effect on myopia. Ophthalmic Physiol Opt. 2015;35:252-62.

57. Hobday R. Myopia and daylight in schools: a neglected aspect of public health? Perspect Public Health. 2016;136:50-5.

58. Li S, Li S, Kang M, Zhou Y, Liu L, Li H, et al. Near Work Related Parameters and Myopia in Chinese Children: the Anyang Childhood Eye Study. PLoS One. 2015;10:e0134514.

59. Sandoval HP, Donnenfeld ED, Kohnen T, Lindstrom RL, Potvin R, Tremblay DM, et al. Modern laser in situ keratomileusis outcomes. J Cataract Refract Surg. 2016;42:1224-34.

60. Alió JL, Toffaha BT. Refractive Surgery With Phakic Intraocular Lenses: An Update. Int Ophthalmol Clin. 2013;53:91-110.

61. Emarah A, El-Helw M, Yassin H. Comparison of clear lens extraction and collamer lens implantation in high myopia. Clin Ophthalmol. 2010;14:447-54. 